3. Multi-resistant organism and Clostridium difficile infection (MDRO/CDI) module. Centers for Disease Control and Prevention website. https://www.cdc.gov/nhsn/pdfs/pscmanual/ 12pscmdro_cdadcurrent.pdf. Accessed February 19, 2018.

4. Moehring RW, Lofgren ET, Anderson DJ. Impact of change to molecular testing for Clostridium difficile infection on healthcare facility associated incidence rates. Infect Control Hosp Epidemiol 2013;34:1055-1061.

5. McDonald LC, Gerding DN, Johnson S, et al. Clinical practice guidelines for Clostridium difficile infection in adults and children: 2017 update by the Infectious Diseases Society of America (IDSA) and Society for Healthcare Epidemiology of America (SHEA). Clin Infect Dis 2018;66:e1-e48.

6. Bartsch SM, Umscheid CA, Nachamkin I, Hamilton K, Lee BY. Comparing the economic and health benefits of different approaches to diagnosing Clostridium difficile infection. Clin Microbiol Infect 2015;21:1-9.

7. Updated guidance on the diagnosis and reporting of Clostridium difficile. United Kingdom National Health Service website. https:// www.gov.uk/government/uploads/system/uploads/attachment_data/ file/215135/dh_133016. pdf. Accessed March 13, 2018.

\section{What Really Works for Scope Reprocessing?}

To the Editor-Duodenoscopes used for endoscopic retrograde cholangio-pancreatography (ERCP) have complex designs that make reprocessing challenging. Infections have been linked to manual cleaning of the scope especially its forceps elevator. Other factors that contribute to infections include use of unsterile water and inappropriate storage of scopes. ${ }^{1,2}$ Despite duodenoscope reprocessing procedures exceeding manufacturer's recommendations, high-concern organisms such as
Klebsiella spp and Pseudomonas spp have been implicated in clinical infections. ${ }^{3,45}$ Media reports of high-concern organisms, such as carbapenem-resistant Enterobacteriaceae (CRE) and extended-spectrum $\beta$-lactamase (ESBL) outbreaks linked to duodenoscopes, have heightened awareness regarding reprocessing procedures. ${ }^{3,4}$ Infections from duodenoscopes have been linked to positive cultures isolated from urine, blood, abscesses, and stool. ${ }^{1,2}$ Mortality associated with contaminated duodenoscopes is $\sim 16 \%$ with all organisms and $56 \%$ with CRE. ${ }^{4}$ These mortality rates emphasize the need for optimal reprocessing practices. The World Health Organization emphasizes teambased collaborations, such as multidisciplinary teams (MDTs), to improve communication among healthcare workers. ${ }^{6}$ Many studies have shown the benefits of MDTs in reducing nosocomial infections like bloodstream infections. ${ }^{7-9}$ Multidisciplinary teams are effective at reducing infection rates through rapid identification of breakdowns in the process. ${ }^{7}$ We studied the impact of creating a MDT with clear roles and real-time huddles to optimize our scope-reprocessing practices.

This retrospective study was conducted at a tertiary-care academic medical center with 401 beds. We aimed to evaluate the impact of an MDT with clear roles on the reprocessing of duodenoscopes. Reprocessing Olympus TJF-Q180V duodenoscopes along with surveillance cultures of the duodenoscope tip (including forceps elevator) were evaluated during the baseline period (January 2016 through June 2016) and during our intervention period (September 2016 through July 2017). An MDT was created in July 2016 composed of representatives from the endoscopy center, the sterile processing department (SPD), the infection prevention department (IP), as well as hospital leadership. We utilized a responsibility assignment matrix (RAM) to outline responsibilities of team members (Table 1). The results of surveillance cultures were grouped based on risk to humans, as defined by Centers for

T A B LE 1. Responsibility Assignment Matrix (RAM) Implemented as a Part of Our Intervention

\begin{tabular}{|c|c|c|c|c|}
\hline Process Name/Description & $\begin{array}{l}\text { GI } \\
\text { Team } \\
\text { Member }\end{array}$ & $\begin{array}{l}\text { CSPD } \\
\text { Team } \\
\text { Member }\end{array}$ & $\begin{array}{l}\text { IP } \\
\text { Team } \\
\text { Member }\end{array}$ & $\begin{array}{l}\text { Hospital Leadership } \\
\text { Team Member }\end{array}$ \\
\hline $\begin{array}{l}\text { Gather scope reprocessing documentation and bring copies to team huddle: } \\
\text { reprocessing log, ATP testing log, patient log sheet, HLD printout, pick up log, culture } \\
\text { collection log, ETO record system, ETO print out. }\end{array}$ & I & $\mathrm{R}$ & $\mathrm{C}$ & I \\
\hline Identify patients involved and bring intraoperative documentation to team huddle. & $\mathrm{R}$ & I & $\mathrm{C}$ & I \\
\hline Determine risk to patients involved. & $\mathrm{R}$ & $\mathrm{R}$ & $\mathrm{R}$ & $\mathrm{R}$ \\
\hline Determine whether patient communication is necessary. & I & I & I & $\mathrm{R}$ \\
\hline $\begin{array}{l}\text { After hours and weekends, CSPD member validates whether HLD requirements } \\
\text { achieved and passed leak test and whether ETO cycle was completed with no errors. } \\
\text { GI member gathers patient information. CSPD and GI members to email complete } \\
\text { investigation to IP within } 2 \text { hours. IP member send out summary report within } \\
1 \text { hour. }\end{array}$ & $\mathrm{R}$ & $\mathrm{R}$ & $\mathrm{C}$ & I \\
\hline
\end{tabular}

NOTE. R, responsible; I, informed; C, consulted; GI, gastroenterology; CSPD, central sterile processing department; IP, infection prevention; ATP, adenosine triphosphate; HLD, high-level disinfection; ETO, ethylene oxide. 
Disease Control and Prevention, as low- and high-concern bacteria. $^{6}$

The reprocessing of duodenoscopes at our center starts with bedside manual cleaning followed by repeat manual cleaning (within an hour) in the sterile processing department. To detect any residual biological material, adenosine triphosphate (ATP) testing is then performed on 5 spots of the duodenoscope: surface, 3 channels, and the elevator. If ATP levels are $<100$ relative light units (RLU), the duodenoscope undergoes high-level disinfection (HLD). If the duodenoscope fails ATP testing, the duodenoscope is recleaned following manufacturer's instructions and undergoes ATP retesting. After HLD with an automated endoscopic reprocesser, a total of 10 duodenoscopes undergo surveillance cultures every month. Duodenoscopes that are cultured are sent through HLD again. All duodenoscopes are then sterilized with ethylene oxide (ETO) prior to use. We have 24 scopes, and $92 \%$ of the scopes undergo surveillance cultures in a span of 4 months. Our monthly surveillance cultures represent efficacy of manual cleaning.

During the baseline period (January 2016 through June 2016), scopes were cultured after $9.4 \%$ of the procedures $(\mathrm{n}=267)$. During the intervention period (September 2016 through July 2017), cultures were obtained after $20.3 \%$ of the procedures $(\mathrm{n}=492 ; P<.05)$. During our baseline period, 10 of 25 cultures were positive $(40 \%)$. During the intervention period, 4 of 100 cultures were positive $(4 \% ; P<.05)$. We reduced our culture positivity by $36 \%$ by increasing the efficacy of our manual cleaning. Culture positivity is the ratio of positive cultures divided by number of scopes cultured. Compliance with the policy for obtaining cultures increased from $41.7 \%$ during the baseline period to $90.9 \%$ during the intervention period. Compliance was defined as a ratio of number of cultures obtained and number of cultures expected to be obtained during a defined period. Our compliance with the policy for obtaining cultures increased by $49.2 \%$. Our compliance with the policy for manual cleaning within 1 hour of bedside cleaning increased from 38.5\% (47 of 122 cultures) in the baseline period to $50.8 \%$ (375 of 738 cultures; $P<.05)$ in the intervention period. Improvement in compliance with other steps in the process was not statistically significant.

By establishing clear responsibilities with RAM (Table 1) and emphasizing real-time huddles (when scope cultures are positive), we reduced the rate of culture positivity significantly from $40 \%$ to $4 \%$. We attribute our success to weekly meetings of MDT members from infection prevention and central sterile processing, which created a high level of engagement. We also developed a process of sending a notification (blast) page to all team members when a scope culture was positive. When a blast page was issued, all team players huddled within hours and conducted a root cause analysis. We used a shared database to track each endoscope from the time of use on the first patient to the subsequent patient. The data included in this database were added by different team members. An action plan was created, and a communication was sent to everyone on the RAM within 24 hours of each meeting.

This study has several limitations. It was a single-center experience, which reduces its generalizability. We did not have a control group, which reduces our confidence that these results were due to the intervention. We relied on capturing compliance based on documentation by personnel for most of the processes in our protocol, which allows for human error.

In conclusion, by emphasizing principles of accountability (RAM) and effective communication (real-time huddles), we were able to show improved efficacy of manual cleaning of endoscopes, which was indicated by reduction in the rate of culture positivity.

\section{ACKNOWLEDGMENTS}

We thank the central processing team and endoscopy center personnel for their contributions to this project.

Financial support: No financial support was provided relevant to this article. Potential conflicts of interest: All authors report no conflict of interest relevant to this article.

\section{Neha Nanda, MD; ${ }^{1,2}$ Preciosa Marasigan, $\mathrm{RN} ;{ }^{2}$ Stephanie Hall, MD; ${ }^{3}$ Evan Mosier, $\mathrm{MD}^{4}$}

Affiliations: 1. Division of Infectious Diseases, Department of Medicine, Keck School of Medicine, University of Southern California, Los Angeles, California; 2. Department of Healthcare Epidemiology and Infection Prevention, Keck School of Medicine, University of Southern California, Los Angeles, California; 3. Keck School of Medicine, University of Southern California, Los Angeles, California; 4. Department of Medicine, Keck School of Medicine, University of Southern California, Los Angeles, California.

Address correspondence to Neha Nanda, MD, Division of Infectious Disease, Department of Medicine, Keck School of Medicine, University of Southern California, 1500 San Pablo, Los Angeles, CA 90030 (neha.nanda@med.usc.edu).

PREVIOUS PRESENTATION. This work was presented as "Impact of Multidisciplinary Team on Duodenoscope Reprocessing to Minimize Infections With High-Concern Organisms" at ID Week 2017 on October 4, 2017, in San Diego, California.

Infect Control Hosp Epidemiol 2018;39:888-890

(c) 2018 by The Society for Healthcare Epidemiology of America. All rights reserved. 0899-823X/2018/3907-0024. DOI: 10.1017/ice.2018.98

\section{REFERENCES}

1. KovalevaJ, Peters FT, van der Mei HC, Degener JE. Transmission of infection by flexible gastrointestinal endoscopy and bronchoscopy. Clin Microbiol Rev 2013;26:231-235.

2. MuscarellaLF. Risk of transmission of carbapenem-resistant Enterobacteriaceae and related "superbugs" during gastrointestinal endoscopy. World J Gastrointest Endosc 2014;6:457-474.

3. EpsteinL, Hunter JC, Arwady MA, Tsai V, et al. New Delhi metallo- $\beta$-lactamase-producing carbapenem-resistant Escherichia coli associated with exposure to duodenoscopes. JAMA 2014;312:1447-1455. 
4. Wendorf KA, Kay M, Baliga C, et al. Endoscopic retrograde cholangiopancreatography-associated AmpC Escherichia coli outbreak. Infect Control Hosp Epidemiol 2015;36:634-642.

5. Aumeran C, Poincloux L, Souweine B, et al. Multidrug-resistant Klebsiella pneumoniae outbreak after endoscopic retrograde cholangiopancreatography. Endoscopy 2010;42:895-899.

6. Framework for action on interprofessional education and collaborative practice. World Health Organization website. http:// www.who.int/hrh/resources/framework_action/en/ Published 2010. Accessed April 10, 2018.

7. Walz JM, Ellison RT, Mack DA, et al. The bundle "Plus": the effect of a multidisciplinary team approach to eradicate centralline-associated bloodstream infections. Anesth Analg 2015;120: 868-876.

8. Gillespie BM, Kang E, Roberts S, et al. Reducing the risk of surgical site infection using a multidisciplinary approach: an integrative review. J Multidiscip Healthc 2015;8:473-487.

9. Miller R, Simmons S, Dale C, Stachowiak J, Stibich M. Utilization and impact of a pulsed-xenon ultraviolet room disinfection system and multidisciplinary care team on clostridium difficile in a longterm acute care facility. Am J of Infect Control 2015;43:1350-1353.

\section{Do Periarticular Joint Injections Present an Increase in Infection Risk?}

To the Editor-Joint replacements are projected to be the most common elective surgical procedures in the coming decade. ${ }^{1,2}$ Effective management of postoperative pain associated with joint replacement surgery improves surgical outcomes by increasing postoperative mobility and reducing the duration of hospitalization. ${ }^{3}$ Many surgeons have recently incorporated local analgesia into pain management regimens for patients undergoing joint replacement surgery. ${ }^{4,5}$ The benefits of using periarticular injections (PAIs) include decreasing opioid consumption and its associated side effects, facilitating earlier mobilization, and decreasing hospital length of stay. ${ }^{6}$ Most PAI techniques involve infiltration of a high-volume, long-acting local anesthetic solution into the surgical incision and surrounding tissues prior to skin closure. A wide variety of medications is used in these injections. ${ }^{7}$ However, based on our hospitals' experiences, these injections frequently include epinephrine, ketorolac, opioids, or steroids.

The Duke Infection Control Outreach Network (DICON) recently identified the use of PAIs as a potential risk for infection during 4 investigations of clusters of SSIs associated with total hip replacements (THRs) and total knee replacements (TKRs). We surveyed hospitals within our network to gather more information about PAI practices. Of 42 hospitals, 20 (48\%) participated in the survey. Of these 20 hospitals, $16(80 \%)$ reported routine use of PAIs in patients undergoing joint replacement surgery. All 16 hospitals used PAIs in patients undergoing TKRs; 14 hospitals also used PAIs in patients undergoing THRs. In 5 hospitals, PAIs included a combination of bupivacaine, morphine, and ketorolac. The remaining 11 hospitals used highly variable combinations of medications. In 12 hospitals, PAIs were prepared in the operating room (OR), while in the other 4 hospitals, PAIs were prepared in the pharmacy. Among the 4 clusters of SSIs we investigated, all prepared PAIs in the OR.

The current literature regarding the relative infection risk associated with periarticular injection use compared to other modalities of pain control is inconclusive. Marques et $\mathrm{al}^{8}$ performed a systemic review and meta-analysis of 2,348 patients undergoing joint replacement surgery (909 THR, 1,439 TKR). ${ }^{8}$ Only eight cases of deep infection requiring surgical debridement or revision occurred in the cohort (overall infection risk, $0.34 \%$ ). In patients with THRs, four deep infections occurred in patients who received PAIs, and one deep infection occurred in the control group (Peto OR 3.47; 95\% CI 0.58, 20.81; $P=0.17$ ). In patients with TKRs, two deep infections occurred in patients who received PAIs compared to one deep infection that occurred in the control group (Peto OR 1.85; 95\% CI 0.19, 17.83; $P=0.59$ ). Thus, the increased number of infections in the PAI groups was not statistically significant. The six patients with deep infection after PAIs received their PAI through a postsurgical catheter that remained in place following surgery.

Jiang et $\mathrm{al}^{9}$ performed a meta-analysis that included an analysis of wound complication rates following TKA and THA in which PAIs were administered. This study showed no statistically significant difference in wound complication rates for either surgery type among patients who received PAIs compared to those who did not. Wang et $\mathrm{al}^{10}$ performed a meta-analysis of ten studies that compared pain control in patients who received PAIs after TKA and patients who received a nerve block. Of these studies, 3 reported that wound complications were not significantly increased in the PAI group (odds ratio, $1.57 ; 95 \% \mathrm{CI}, 0.40-6.16 ; P=.52)$. Although the preceding studies suggest that rates of infection-related complications in patients undergoing PAIs are low, most of the previous studies were underpowered or included an inadequate duration of follow-up to detect an association between PAI and SSI risk.

From a theoretical and practical perspective, the use of PAIs has numerous potential risks for the introduction of bacteria into the joint space or incisional tissues. For example, PAIs are usually compounded in the OR without the use of a sterile hood. Furthermore, OR personnel who lack formal training in drug compounding are typically responsible for preparing these injections. Bacteria may also be introduced through the skin at the time of drug injection, especially if 